\title{
e-Migrinter
}

17 | 2018

Enseigner les migrations internationales

\section{Didi-Huberman, Georges ; Giannari, Niki (2017) Passer quoi qu'il en coûte}

\section{Oriana Philippe}

\section{(2) OpenEdition}

\section{Journals}

Édition électronique

URL : https://journals.openedition.org/e-migrinter/1215

DOI : 10.4000/e-migrinter.1215

ISSN : 1961-9685

Éditeur

UMR 7301 - Migrinter

\section{Référence électronique}

Oriana Philippe, « Didi-Huberman, Georges ; Giannari, Niki (2017) Passer quoi qu'il en coûte », eMigrinter [En ligne], 17 | 2018, mis en ligne le, consulté le 20 mai 2021. URL : http://

journals.openedition.org/e-migrinter/1215; DOI : https://doi.org/10.4000/e-migrinter.1215

Ce document a été généré automatiquement le 20 mai 2021.

Tous droits réservés 


\title{
Didi-Huberman, Georges ; Giannari, Niki (2017) Passer quoi qu'il en coûte
}

\author{
Oriana Philippe
}

\section{RÉFÉRENCE}

Didi-Huberman, Georges ; Giannari, Niki (2017) Passer quoi qu'il en coûte, Paris, les Éditions de Minuit, 98 p.

1 La bibliothèque du laboratoire Migrinter ne cesse d'étoffer sa collection par des supports et sujets variés ou des approches disciplinaires nouvelles. L'acquisition de l'ouvrage de Georges Didi-Huberman et Niki Giannari nous offre une lecture extrêmement précieuse, un voyage entre philosophie, littérature, art et sciences humaines. Elle est déconcertante aussi cette lecture, presque nécessaire à qui est toujours soucieux du travail d'objectivation, d'analyse des faits, de justification des preuves. Les deux auteurs nous livrent un cri de colère, un pamphlet qui dénonce le traitement infligé aux migrants par l'Europe. L'œuvre n'a effectivement pas vocation à réaliser une analyse objective et détachée. Ce n'est pas un ouvrage de documentation ou un rapport de situation. L'ouvrage part du postulat que le lecteur sait, l'objectif n'est pas d'informer sur les faits, de décrire ou d'expliquer. L'ouvrage est parcouru de tristesse et condamne fermement une histoire qui se répète, une inhumanité impardonnable.

2 Idomeni, mars 2016. Environ " treize mille personnes fuyant les guerres de Syrie, d'Afghanistan et d'ailleurs, tentent de passer la frontière gréco-macédonienne " laquelle est « justement en train de se refermer devant eux. » (p. 27)

3 C'est ici que tout commence. Niki Giannari s'affaire à Idomeni pour améliorer un tant soit peu les conditions de vie à la frontière. Elle y est rejointe par son amie Maria Kourkouta et ces dernières décident de réaliser un documentaire qui s'intitulera "Des spectres hantent l'Europe ». Niki Giannari écrit alors un poème qui fera partie de l'animation sonore de ce témoignage cinématographique. Ce poème, ce sera la matrice 
de cet ouvrage de Georges Didi-Huberman. Il constitue la première partie de l'ouvrage où il est présenté traduit en français, mais aussi en grec, dans sa version originale. À ce titre, ce n'est pas Niki Giannari qui a qualifié de poème son enchevêtrement de mots. Cela a été un choix de Georges Didi-Huberman. Elle, elle l'appelle Lettre de Idomeni. Elle dit que ce ne sont que des «bribes destinées à se caler sur les images du film, rien de plus. Ce ne sont que des phrases, adressées, depuis Idomeni, à un ami européen.» (p. 29)

4 L'analyse de Georges Didi-Huberman constitue la deuxième partie de l'ouvrage, intitulée "Eux qui traversent les murs ». Cette deuxième partie est ponctuée de petits chapitres, consacrés chacun à un vers différent du poème et source de sa réflexion. L'auteur y a sélectionné huit passages ${ }^{1}$ qui constitueront la colonne vertébrale de son développement. Il les a choisis sans règle explicite. Il ne les place pas dans leur ordre d'apparition, il en choisit plusieurs dans une partie aucun dans une autre. Il semble avoir suivi ce qui l'inspirait, ce qui l'animait le plus. Pour chaque syntagme, il emporte le lecteur dans une réflexion approfondie, inspirée par l'épigramme de Niki Giannari et enrichie par ses «incitations». De même, il évoque à plusieurs reprises le documentaire. L'auteur décortique, les supports mais aussi les mots, par un travail profond sur l'étymologie et les liens entre les termes.

5 La structure légère et épurée offre une certaine liberté aux pensées de l'auteur qui peuvent se déployer sans limite. Cette liberté dans la forme se retrouve à plusieurs reprises, des choix inédits permettant une meilleure mise en lumière de son travail de pensée.

6 Le texte est animé de plusieurs thématiques transversales, de la récurrence de plusieurs auteurs et de certains outils qui viennent nous rappeler la continuité du raisonnement. Ainsi, si parfois on a l'impression de s'éloigner de notre trame, d'être parti bien loin d'Idomeni, on réalise ensuite que le tissage est bien noué, avec ses rappels horizontaux, verticaux, diagonaux qui jalonnent le texte. Du début à la fin de l'ouvrage, Idomeni est le centre du monde, de l'Europe, de l'histoire malgré toutes ces extensions géographiques et historiques.

7 La partie « Eux qui traversent les murs » commence par un portrait de Niki Giannari (pp. 23-28). L'auteur réalise une description affectueuse d'une personne qu'il semble non seulement apprécier, mais également admirer. La présentation de Niki Gianniri ne se veut pas strictement informative : le texte révèle, en réalité, peu d'information technique à son sujet. L'auteur dresse un portrait, attaché, chaleureux, émerveillé, personnel, et presque intime. Le lecteur se sent presque de trop entre les deux concernés. En fait, dès les premières pages du livre, Georges Didi-Huberman balaye toute vocation d'objectivité à son ouvrage. Niki Giannari est « la plus clandestine, sans doute, des écrivaines grecques aujourd'hui. Elle interroge en chacun le désir et le nondit» (p.25) elle possède une "inquiétante [...] générosité fondamentale. [...] Sa puissance est celle, dialectique, maïeutique, de la parole et de la pensée vivantes » (p. 26).

8 Cette biographie s'intitule justement «Pour autrui » car aux yeux de Georges DidiHuberman, cette femme a en elle la " passion pour autrui» (p. 26). Cette même passion qui « la ferait presque disparaître » (ibid.) malgré sa puissance. En outre, ce témoignage cinématographique de Niki Giannari et Maria Kourkouta est pour autrui. Pour celui qui a vu et vécu mais n'a pas été en mesure de témoigner; et pour celui qui n'a pas pu ou voulu voir. « Le témoignage se tient donc entre deux autruis » (p. 28). Le témoignage est 
une des thématiques piliers abordées par Georges Didi-Huberman. L'œuvre de Niki Giannari, son texte, mais aussi le documentaire réalisé avec son amie, sont des actes de témoignages aux yeux de l'auteur du livre.

On témoigne de ce qui s'est passé. Cette réflexion ne nous laisse donc pas à Idomeni en mars 2016. Au contraire, l'auteur nous transporte dans le temps et dans l'espace grâce à une richesse bibliographique stupéfiante. Au-delà de l'histoire du camp d'Idomeni, c'est dans l'histoire de l'humanité que Georges Didi-Huberman nous invite à voguer. Déjà par son épigramme, Niki Giannari nous rappelle l'histoire et notamment celle de la Seconde Guerre mondiale : « Les hommes vont oublier ces trains-ci, comme ces trains-là ${ }^{2}$ » (Vers 2 et 3, p. 11), " Portbou, 26 septembre de l'an 1940, le jour où la frontière s'est fermée, Walter Benjamin s'est donné la mort » (Vers 59 et 60, p. 15), allusions dont va se saisir amplement Georges Didi-Huberman. À cet effet, il va prendre intensément appui, tout au long de son texte ${ }^{3}$, sur une autre figure féminine importante aux mots poignants, Hannah Arendt, qu'il nous invite explicitement à relire. Nous oscillons entre ces deux femmes, qui chacune à son époque, ont témoigné de ce qui ne devait être gardé sous silence. « C'est là une situation proprement - éthiquement - inhumaine. À Idomeni, Niki Giannari a bien compris, comme Hannah Arendt l'avait fait ailleurs en Europe, qu'il était très facile d'ajouter de l'inhumanité à l'inhumanité » (p. 47).

Niki Giannari et Georges Didi-Huberman martèlent leurs textes de rappels et de parallèles à l'histoire de la Shoah, comme pour nous imposer de voir que ce qui a un jour été qualifié du summum de l'inhumanité se reproduit devant nos yeux. Un de ces parallèles est l'histoire de Walter Benjamin, « dont la figure traverse, en réalité, tout ce poème » (p. 29), mais aussi tout cet ouvrage ${ }^{4}$, ce réfugié qui se donna la mort à la frontière espagnole car il lui était refusé de passer. C'est aussi l'histoire de Heinrich Heine (pp.69-71). Georges Didi-Huberman rappelle qu'il est des tragédies pour lesquelles l'oubli est impossible. «Mais la cendre / se souvient » scande Niki Giannari (Vers 4 et 5, p. 11).

11 Cette inhumanité inoubliable, c'est un autre des piliers transversaux qui fondent cet ouvrage. À Idomeni, l'inhumanité se traduit - entre autre - par l'empêchement de passer. La seule chose qu'ils demandent, passer. «Passer. Passer quoi qu'il en coûte. Plutôt crever que ne pas passer, [...] Passer pour ne pas mourir dans ce territoire maudit et dans sa guerre civile, [...] passer pour vivre comme sujets du droit. » (p. 39). Ils sacrifient tout pour cela, pour tourner le dos à la mort. "On humilie les réfugiés lorsqu'on exige d'eux qu'ils n'exigent rien du tout.» (p. 37) Le seul accueil que nous sommes capable de leur offrir c'est de les "parquer» dans des camps. Georges DidiHuberman explore cet espace, cette notion de camp sous différents angles. Tout d'abord, il évoque sa naissance par la création du fil barbelé (Olivier Razac) (p. 37) et l'indignation de Hannah Arendt en 1943 face à cette " "humanité parquée" à laquelle sont réduites les personnes dites "déplacées" » (p. 38). Il poursuit ensuite cette analyse de la notion de camp en s'inspirant des travaux de Giorgio Agamben (p. 38), Gilles Lhuilier, Olivier Lecour Grandmaison, Alain Rey, Claire Rodier (pp. 41-42) et Michel Agier (p. 52). Ainsi l'auteur remarque : "Ils ont risqué leur vie pour gagner un espace de droit. Aujourd'hui un camp les accueille, leur donne à manger, leur fournit une tente ou un vêtement imperméable. Mais c'est un espace où l'on continue d'attenter à leur vie civique. On les condamne à l'attente bureaucratique pour connaître leur "statut". Ils font la queue, dans l'attente d'être humains. Pourtant ils sont bien là, devant nous, avec leurs gestes, leurs visages, leurs paroles. » (p. 39) 

deux sont interdépendants. Alors nous sommes directement impliqués. Ce point, c'est un élément fondateur pour la militante et pour le philosophe : l'histoire de ces spectres à la frontière, c'est notre histoire à nous. En effet, des spectres hantent l'Europe, c'est le titre choisi pour le documentaire et pour le premier chapitre. Le choix du vocable « spectre » n'est pas anodin. Georges Didi-Huberman choisit, tout au long de son écrit, de faire usage d'une variation graphique pour mettre en avant certaines lexies. En effet, la plume soignée et magnifique de l'auteur danse entre jeux-de-mots et réflexions sur l'étymologie et la signification des mots. En l'occurrence, il fait usage du style italique pour mettre en lumière certains termes. Ces mots accompagnent souvent le vers en cours de développement. Ainsi, dans cette partie "Des spectres hantent l'Europe ", sont présentés en italique les mots suivants : « devenir fantômes ", « êtres d'ailleurs », « revenir », « parents revenants », « peuples au pluriel », « être ancestral» (pp. 31-32). Ces termes portent un message : nous voulons oublier que ces réfugiés sont nos propres fantômes, nos propres parents. "Après tout les réfugiés ne font que revenir. [...] nous sommes tous les enfants de migrants » (p. 31).

13 Alors ce livre c'est aussi une diatribe sur nous-mêmes, peuples d'Europe. Nous bafouons l'humanité de nos propres parents. Or, « la question n'est pas vraiment de savoir si les "spectres de l'Europe" sont ceci ou cela - juifs, païens, Byzantins, communistes, musulmans, parias colonisés ou que sais-je encore. C'est plutôt celle de savoir pourquoi l'Europe produit des spectres. Pourquoi elle tente d'oublier quelque chose qui lui revient pourtant fondamentalement - qui la regarde et la concerne, passe en elle, la traverse - comme revenance et retour du refoulé»(p. 71). Les deux auteurs se questionnent alors sur nous-même, avec notamment à l'appui, Freud - le «malaise dans la culture » aurait donc, pour l'une de ses composantes essentielles, une hostilité envers l'autre (p.62), Marx et Engels, - le titre du documentaire «consonne évidemment [...] avec la phrase inaugurale du Manifeste communiste:" Un spectre hante l'Europe " " (p. 32) ou encore Hannah Arendt qui constate « le déclin de l'État-nation et la fin des droits de l'homme » (p. 44).

Et pourtant, ceux auxquels nous ne sommes pas en mesure d'offrir une humanité décente, nos spectres, nos fantômes, sont pleins de force, d'espoir, de volonté, d'envie de vivre. Une force à envier pour Niki Giannari « Qu'ils m'emmènent avec eux/ eux qui savent encore être en mouvement»(p.17). Et si Hannah Arendt affirmait que les réfugiés représentaient l'avant-garde de leur peuple, Georges Didi-Huberman ajoute qu'ils sont aussi l'avant-garde des peuples qui les accueillent (p. 84). Leur volonté de passer est si forte que Niki Giannari constate : «Je comprends / qu'ils sont déjà passés » (p. 21). Morts ou vivants, ils passent. C'est la survivance (p.61). Or cette survivance constitue la clef du témoignage. Et il faut témoigner. À tout prix et par tout moyen. Un de ces moyens, c'est l'image. L'« image visuelle ou poétique [...] remonte le temps [...] et se rend capable » (p.60) de témoigner. Ce pouvoir est essentiel, il est évoqué dans les proses de Paul Celan sur lesquelles s'ouvre le livre, annonciateur du message à venir : «Avec la fatalité des images et leur contre-fatalité [...] attend, cristal de souffle, ton inébranlable témoignage [...] vers nous et loin de nous et vers nous » (p. 7 et 57). Il est mentionné aussi par Pline l'Ancien, lequel distinguait l'image au sens propre, l'« imago " comme constituant "un opérateur de dignité[...] utilisé comme un objet de transmission généalogique, c'est-à-dire de restitution et de passage » (p. 82). Et enfin, «Aby Warbug [qui] comprenait l'histoire des images comme une "histoire de fantômes 
pour grandes personnes": une histoire où les images se montrent capables de "revenir" [...] cette puissance-là, Warburg avait choisi de la nommer survivance [...] la capacité [...] de passer à travers temps. » (p. 60)

Georges Didi-Huberman relève les nombreuses expositions de photos sur les frontières et les camps qui les jouxtent. "Toutes ces productions d'images [...] n'y dessineraientelles pas comme un immense monument d'accusation porté contre nos propres gouvernements? » questionne-t-il (pp. 81-82). Lui-même ponctue son texte de quelques photos en noir et blanc. Pas de voyeurisme, pas de pathétisme malgré la gravité de la situation à Idomeni, mais des images qui témoignent. Des images du documentaire aussi, avec les bribes de Niki Giannari en sous-titre, pour redoubler leur puissance, permettant au lecteur de se représenter l'effet donné dans le documentaire.

Sur ces photos d'Idomeni, de nombreux enfants. Un choix précis, car ces enfants, ce seront les premiers témoins. Ces enfants "têtus» qui "se donnent émus à la vie " (p. 17) porteront le témoignage de l'inhumanité à laquelle ils ont assisté. Aujourd'hui, ce sont des « orphelins, épuisés / ayant faim, ayant soif» (Vers 28 et 29, p. 13). Demain, ils engendreront eux-aussi le peuple d'Europe. "L'enfance actualise la survie et l'héritage du genre humain " (p. 85). Il est donc impossible d'oublier car l'humanité d'aujourd'hui engendre l'humanité de demain. «La cendre se souvient » (Vers 4 et 5, p. 11).

17 Georges Didi-Huberman achève son ouvrage sur ses «Incitations" (pp. 91-99) où il mentionne ses références littéraires. Les auteurs intitulent habituellement cette liste Bibliographie. Mais, en réalité, ce terme incitations est bien plus approprié pour un ouvrage qui emporte le lecteur dans un voyage littéraire, artistique et réflexif sans limite; pour un ouvrage qui ne cherche pas à nous informer mais à nous faire réfléchir et méditer ; pour un ouvrage, enfin, qui déverse à nos pieds de multiples éclipses bibliographiques telles une invitation à aller plus loin, à découvrir et à approfondir. Alors oui, toutes ces mentions sont des incitations plus que des ressources bibliographiques. Elles ont inspiré et incité l'auteur et entendent inspirer et inciter le lecteur.

18 Ne vous attendez pas à un livre qui rentre dans une catégorie. Il fait partie de ceux qui franchissent les limites, de ceux qui passent, quoi qu'il en coûte.

\section{NOTES}

1. Les 8 passages du poème de Niki Giannari choisis par Georges Didi-Huberman pour intituler ses chapitres sont: Des spectres hantent l'Europe, Une fois encore / tu ne peux te poser nulle part, Ne demandent rien / seulement passer, Un jour avant / ou un jour après, Mais la cendre / se souvient, Orphelins, épuisés / Séculaires et sacrés, Ils passent / et ils nous pensent, Qu'ils m'emmènent avec eux / eux qui savent encore être en mouvement.

2. À ce titre, Georges Didi-Huberman éclaire ce vers par les propos suivants: "Quand Niki Giannari nous parle de "spectres qui hantent l'Europe", on a envie de lui demander : mais depuis où exactement viennent-ils hanter l'Europe? Et surtout: depuis quand? Une première 
réponse est donnée, dès les premières lignes de son texte, à travers la mise en relation entre «ces trains-ci - qu'en 2016 elle a vus passer en bordure du camp d'Idomeni, trains de marchandises à destination du nord de l'Europe - et « ces trains-là » ... Quels trains-là » ? Évidemment les trains qui, partant de Thessalonique vers le nord, auront convoyé vers les camps d'extermination nazis, peut-être sur cette même ligne de chemin de fer, près de quatre-vingt-huit pour cent de la population juive de cette ville, soit environ cinquante-quatre mille personnes. » (p. 48)

3. Essentiellement dans les trois premières parties mais ses écrits sont également repris plus loin dans l'ouvrage.

4. Son histoire se retrouve dans les parties « Des spectres hantent l'Europe », « Un jour avant, un jour après » "Mais la cendre se souvient ", "Orphelins, épuisés, séculaires et sacrés ", "Qu'ils m'emmènent avec eux, eux qui savent encore être en mouvement ».

\section{AUTEURS}

\section{ORIANA PHILIPPE}

Doctorante en Géographie, Laboratoire Migrinter (UMR 7301) CNRS / Université de Poitiers

Oriana.philippe@univ-poitiers.fr 